\title{
ALGUNAS OBSERVACIONES SOBRE LA DÉCADA DE LA EDUCACIÓN AMBIENTAL PARA EL DESARROLLO SOSTENIBLE Y LA TAREA PENDIENTE DE LA EDUCACIÓN AMBIENTAL SUPERIOR
}

\begin{abstract}
Resumen
El objetivo de este artículo es presentar y discutir el contexto de la Década de la Educación Ambiental para el Desarrollo Sostenible, de las Naciones Unidas, señalando las dificultades del concepto de desarrollo sostenible en sí mismo, para demostrar que el énfasis de la educación ambiental superior debe girar en torno a otras preguntas, como ¿En qué ha fallado la formación de los profesionales que no logran comprender o asumir la responsabilidad de la complejidad ambiental? ¿Por qué los profesionales universitarios no reconocen la fragilidad de la vida en sus decisiones laborales cotidianas? Para responder a estas preguntas el texto se plantea el problema desde el pensamiento y la epistemología ambiental. Por lo tanto la educación ambiental superior no se trata de una educación que provea información sobre legislación, instrumentos de gestión ambiental, etc., sino que tiene como objetivo formar futuros profesionales con criterios epistemológicos y teóricos amplios capaces de comprender la complejidad ambiental.
\end{abstract}

María Luisa Eschenhagen ${ }^{1}$

Palabras-claves: Educación ambiental superior. Pensamiento y epistemología ambiental. Desarrollo sostenible.

\section{SOME OBSERVATIONS ON THE DECADE OF ENVIRONMENTAL EDUCATION FOR SUSTAINABLE DEVELOPMENT AND THE REMAINING TASK OF UNIVERSITY ENVIRONMENTAL EDUCATION}

\begin{abstract}
The goal of this article is to present and discuss the context of the Decade of Environmental education for Sustainable Development of the United Nations, pointing out the difficulties of the concept "sustainable development" itself, to demonstrate that the emphasis of the environmental education in universities must be around questions as: in what issues has failed the education of professionals which are not able to understand or assume the responsibility of the environmental complexity? Why university professional's don't recognize the fragility of life in their daily labor decisions? To respond this questions the text consider the problem from the environmental epistemology and thought. That's why environmental education in universities is not about an education which provides information about legislation, instruments of environmental management, etc. but has as goal to educate future professionals with wide epistemological y theoretical judgment, able to understand the environmental complexity.
\end{abstract}

Keywords: Environmental education in universities. Environmental thought and epistemology. Sustainable development.

${ }^{1}$ Profesora investigadora de la Universidad Pontificia Bolivariana, sede Medellín, Colombia. Grupo Territorio, www.pensamientoambiental.de. 


\title{
ALGUMAS OBSERVAÇÕES SOBRE A DÉCADA DA EDUCAÇÃO AMBIENTAL PARA O DESENVOLVIMENTO SUSTENTÁVEL E A TAREFA PENDENTE DA EDUCAÇÃO AMBIENTAL UNIVERSITÁRIA
}

\begin{abstract}
Resumo
O objetivo deste artigo é apresentar e discutir o contexto da Década da Educação Ambiental para o Desenvolvimento Sustentável, conforme proposição das Nações Unidas, de las Naciones Unidas, assinalando as dificuldades relativas ao conceito de desenvolvimento sustentável, para demonstrar que a ênfase da educação ambiental em nível superior deve focar em outras perguntas, como: Em que há falhado a formação dos profissionais que não conseguem compreender ou assumir a responsabilidade referente à complexidade ambiental? Por que os profissionais universitários não reconhecem a fragilidade da vida em suas decisões de trabalho cotidianas? Em busca de respostas para tais questões, este texto se embasa no pensamento e na epistemologia ambiental, depreendendo-se, então, que a educação ambiental superior não consiste numa educação para prover aporte de informações sobre legislação, instrumentos de gestão ambiental etc., porém, seu objetivo é a formação de profissionais com critérios epistemológicos e teóricos que os tornem capazes de compreender a complexidade ambiental.
\end{abstract}

Palavras-chave: Educação ambiental superior. Pensamento e epistemologia ambiental. Desenvolvimento sustentável.

\section{Introducción - Contexto}

El tema al que se convocó en el VIII Encuentro de Investigación en Educación Ambiental, en Río de Janeiro, en julio de 2015, es reflexionar sobre Lo no dicho en la década de las Naciones Unidas de la educación para el desarrollo sostenible, y mi reflexión será desde una perspectiva crítica, tanto desde el contexto sociopolítico como desde la educación ambiental superior.

Quiero, primero, señalar que esta revisión se da dentro de una época en la cual ser político o tener posiciones ideológicas políticas claras ya no es deseable o bien visto, o no es posible porque las líneas diferenciadoras entre la izquierda y la derecha se han borrado cada vez más; por lo tanto, ser de un partido u otro ya no tiene mayor relevancia. El sistema económico neoliberal ha impuesto, a nivel global, una visión casi homogenizada que considera que los instrumentos utilizados, como por ejemplo la gestión ambiental, son políticamente neutrales, o que las discusiones ideológicas no tienen nada que ver con discusiones financieras, las cuales deben ser puramente técnicas y de cumplimiento de los acuerdos ${ }^{2}$.

Si hace unos 100 años había unos proyectos sociales claros por los cuales luchar, con ideas claras de cómo organizarse social y económicamente, para bien o para mal, pero los había. Sin embargo, lo que se observa hoy, a nivel mundial, es una gran inconformidad que se expresa ya sea a través de los movimientos de Occupy Wall Street, los indignados en España o la primavera árabe, ya sea a través de las grandes tragedias de los refugiados africanos en el mar mediterráneo, en Myanmar, de cientos de miles de personas de la comunidad minoritaria Rohingya ahogándose en los mares, o de los casi ya millones de migrantes que llegan a Europa. Todos los movimientos de personas - los que protestan y los que huyen, sea por condiciones de guerra o económicas - buscan una vida mejor, más digna y en paz. No obstante, llama la atención que en pocas partes, por no decir en ninguna, ni los políticos o los medios de

\footnotetext{
${ }^{2}$ Un buen ejemplo de ello fue el manejo de la crisis financiera griega, a comienzos del 2014, cuando los ministros de finanzas europeos insistían en mantener las negociaciones en un marco técnico para cumplir acuerdos, señalando que las discusiones ideológicas estaban fuera de lugar.
} 
comunicación, se cuestionan las causas políticas y económicas del sistema-mundo hegemónico, ni mucho menos se vislumbran propuestas concretas para proponer otros proyectos sociales, alternos, sustentables, diversos, viables a largo plazo.

Es esa despolitización la que, por un lado, no permite reconocer las causas, en este caso de los problemas ambientales, y por el otro, la que lleva a caer en trampas discursivas como el desarrollo sostenible. A la despolitización de los discursos también se suma la falta de formación universitaria crítica y reflexiva, lo que impide que los futuros profesionales comprendan y reflexionen sobre la complejidad ambiental cuando son ellos quienes tendrán que tomar toda índole de decisiones en las sociedades, en este caso, sin criterios ambientales. Este es el panorama en el que surge la tarea y deuda que tiene la educación ambiental superior.

Sin reconocer las causas de los problemas ambientales resulta difícil, y hasta imposible, evaluar si una medida como la educación para el desarrollo sostenible resulta ser buena o suficiente. Por lo tanto, es pertinente primero reflexionar sobre qué se entiende sobre problemas ambientales para, luego, identificar cuáles podrían ser sus causas.

Al plantear la primera pregunta, si existen problemas o crisis ambientales, o ecológicos, comienzan las confusiones. Resulta difícil hablar de crisis ecológica si por ecología se entiende una ciencia que estudia las relaciones entre lo biótico y abiótico. Igual que en las matemáticas o la química, la ecología puede tener una crisis como ciencia, una crisis en sus suposiciones, propuestas y lecturas teóricas entre sus comunidades científicas y de ciertos paradigmas establecidos hasta el momento, pero no una crisis, como se entiende comúnmente, como destrucción, contaminación o degradación del entorno físico. Mientras que si por ambiente se entiende la relación que existe entre el ser humano y su entorno físico, la naturaleza, entonces la crisis, el problema, se refiere a la relación que se establece entre los dos, que como consecuencia, efectivamente, tiene la destrucción, contaminación o degradación del entorno físico.

Por lo tanto, el problema ambiental no es el calentamiento global o el territorio deforestado, la pérdida de biodiversidad en sí mismos, entre otros. Estos resultan ser más bien los efectos físicos de una forma específica de relación que se justifica y legitima a través de cosmovisiones, conocimientos, símbolos que median la relación entre ser humano y la naturaleza. Esta relación entre el ser humano y la naturaleza se expresa, en algunos casos, en altas capacidades de adaptación al medio (p.ej. a medios tan agrestes como las culturas de los Inuit, en Groenlandia, los Tuareg, en el Sahara, o los Shuar, en el Amazonas) o se expresa en altas capacidades de transformación sin precedentes del territorio que generan impactos insostenibles e irreversibles sobre los ecosistemas (p.ej. agroindustria y megaminería a cielo abierto).

La segunda pregunta que surge, entonces, es ¿cómo comprender la relación ser humano - naturaleza? Hay muchas formas de comprender esta relación, ya sea desde Descola (2012), quien nos habla de cuatro formas de relacionamiento en su libro "Más allá de la cultura y la naturaleza”, que son los naturalistas, animistas, analogistas y totemistas. La cultura occidental moderna es naturalista, se caracteriza por considerar que existe una diferencia entre seres humanos y el resto de su entorno natural por tener alma y razón. Esta suposición básica implica tener, a la vez, unas concepciones muy específicas del mundo que se expresan a través las concepciones ontológicas y epistemológicas que fue posibilitando esta cultura occidental moderna. Y es a partir de esta suposición básica que puede comprenderse la relación establecida a través de diferentes tipos de mediaciones, ya sea tomando como mediación principal para explicar la relación, la cultura, como lo hace Augusto Ángel Maya (1995, 1996), ya sea el trabajo, en el caso de Guido Galafassi (2001, 2002), o el conocimiento, como lo plantea Enrique Leff (2004, 2006).

Desde mi perspectiva, parece más convincente interpretar la mediación a través de las formas de conocer, es decir cómo se posibilita y construye conocimiento (epistemología). 
Formas de conocer que justifican y legitiman cómo apropiarse del entorno y transformar el territorio, conocimiento que, en últimas, determina también la cultura y las formas de trabajo. No es lo mismo actuar bajo una racionalidad instrumental y economicista, en este caso marcada por un conocimiento epistemológico positivista que no reconoce la complejidad ambiental, que actuar bajo una racionalidad ambiental basada en un conocimiento de la complejidad, diferencia y otredad, como la plantea Leff. De ahí que Leff señala que la crisis ambiental es apenas un síntoma más de la crisis civilizatoria moderna, porque sus formas de conocer, que organizan las estructuras sociales y los modos de producción, resultan ser profundamente insustentables a largo plazo. Esto se termina expresando p.ej. en el calentamiento global, el cual es simplemente el efecto del uso excesivo de energía en la producción económica masiva para el capital, que no reconoce ni la entropía, ni los límites en sus suposiciones teóricas básicas, para justificar y fomentar este tipo de producción.

A partir de esta línea argumentativa, el saber ambiental implica, entonces, visibilizar la racionalidad insustentable y la epistemología que fundamenta y justifica esa producción y consumo sin fin (p.ej. de la producción de carros, de celulares último modelo), así como las relaciones de poder en el saber que le subyacen (LEFF, 2006). Me estoy refiriendo a teorías económicas neoliberales, políticas hegemónicas, sociedades de consumo etc. En este espacio no puedo profundizar, pero sí señalar la dificultad histórica y demostrada que tienen las ciencias sociales, como la economía, la política, la sociología, para pensar y concebir, dentro de sus teorías, la naturaleza (ver ESCHENHAGEN 2012, 2015). Es dentro de este contexto en el que se evidencia la importancia de la epistemología ambiental.

Para poner un ejemplo, una cosa es considerar la dimensión ambiental como un simple factor más dentro de la economía clásica y otra, la economía ecológica. En la primera se reconoce el problema ambiental como una externalidad, una cosa/objeto, que debe ser incorporada dentro de las racionalidades ya establecidas del mercado, como bien lo hace la economía ambiental. Este enfoque simplemente aplica las teorías neoliberales a los recursos naturales para que el mercado regule los problemas ambientales. Desde una dimensión ambiental imbuida por la economía clásica se justifica la existencia, por no decir invención, de los mercados de carbono, de los bienes y servicios ambientales, los mecanismos de producción limpia etc. La segunda, la economía ecológica, considera la entropía y reconoce diferencias entre energías endosomáticas y exosomáticas, y llama la atención sobre el flujo de materia y energía en los ciclos de producción, con lo cual el ciclo necesariamente es abierto y no cerrado como lo plantea la economía clásica ${ }^{3}$. Así, entre las dos teorías existen diferencias ideológicas y epistemológicas claras, con consecuencias diferentes en la forma de relacionarse con el entorno, de transformación del territorio, de posibilitar la vida a largo plazo o no (ver p.ej.: GRANATO; NAHUEL; PENELA, 2009; CAVALCANTI, 2004; CORTÉS LANDÁZURY, 2008).

Es entre estas posiciones diferentes en las cuales entra ahora la discusión en torno al desarrollo sostenible. Esta propuesta política no se puede entender sin considerar el discurso del desarrollo, como lo ha planteado ESCOBAR (1996), y sin considerar la clara decisión política internacional de no seguir fomentando el concepto de ecodesarrollo, concepto que, en medio de la guerra fría, tuvo un tinte, considerado por las directivas de las Naciones Unidas como excesivamente socialista (ver ARIAS, 2003). Por lo tanto, para sustituir el concepto de ecodesarrollo, se instauró el desarrollo sostenible, según el Informe Brundtland (1987), el cual pone las bases teóricas para este nuevo concepto. Es de notar que este informe tiene una perspectiva economicista muy patente, con unas bases políticas y económicas liberales muy

${ }^{3}$ Aunque es de señalar que también esta puede terminar siendo cooptada, del discurso hegemónico del desarrollo, como una forma más sofisticada para poner precios p.ej. a los servicios ambientales. 
claras, e invoca, sin duda alguna, a seguir creciendo, pero ahora sí, considerando el medio ambiente.

Los Estados asegurarán que se trate la conservación como parte integrante de la planificación y ejecución de las actividades de desarrollo y proporcionarán asistencia a otros Estados, en especial a países en desarrollo, en apoyo de la protección del medio ambiente y el desarrollo duradero (BRUNDTLAND, 1987, p. 382).

Es decir, conservar para seguir el desarrollo, en ninguna parte critica realmente el crecimiento económico, ni el sistema económico hegemónico imperante, sino más bien su mal funcionamiento y los problemas que tiene. Se puede leer en el informe:

\begin{abstract}
La espiral descendente de pobreza y degradación medioambiental constituye una pérdida de oportunidades y recursos. Se trata, en particular de una pérdida de recursos humanos. Estas vinculaciones entre la pobreza, la desigualdad y la degradación medioambiental forman un tema importante en nuestro análisis y recomendaciones. Lo que se necesita ahora es una nueva era de crecimiento económico, un crecimiento que sea poderosa a la par que sostenible social y medioambientalmente (BRUNDTLAND, 1987, p.13, subrayado nuestro).
\end{abstract}

Los Estados mantendrán los ecosistemas y los procesos ecológicos indispensables para el funcionamiento de la biosfera, conservarán la diversidad biológica y observarán el principio del óptimo rendimiento sostenible en la utilización de los recursos naturales vivos y de los ecosistemas. (BRUNDTLAND, 1987, p.381, subrayado nuestro)

Por otro lado, la Agenda 21, de Río de 1992, simplemente es la instrumentalización del Informe Brundtland, que señaliza el camino a seguir para lograr ese desarrollo sostenible. Pero comienzan las confusiones, cuando en muchos espacios de gestores políticos, Ongs, académicos etc., se cita, por lo general de manera relativamente descontextualizada, la famosa definición del desarrollo sostenible, sin considerar su contexto económico específico, y se elaboran un sinfín de acomodamientos, con frecuencia acríticos, del concepto, con el fin de obtener financiación para proyectos o aval político, por el political correctness.

A partir de estas discusiones y diferenciaciones es que en español (porque en inglés o alemán no es posible esta diferenciación) los grupos y académicos comenzaron a reconocer esta cooptación del tema ambiental por el discurso hegemónico del desarrollo, y empezaron a formular la conceptualización del desarrollo sustentable. Buena parte de los años 90, la comunidad académica, especialmente latinoamericana, se dedicó a diferenciar estos dos conceptos, que claramente tienen objetivos políticos, económicos y ambientales diferentes. Tal vez donde más cristalizó la diferencia fue en la Carta de la Tierra (2000), elaborada por las Naciones Unidas, con las posiciones del Norte, que aboga por el desarrollo sostenible y $E l$ manifiesto por la vida por una ética para la sustentabilidad (2003), elaborado por los ministros de ambiente latinoamericanos, que reivindica más bien una ética y una sustentabilidad de la vida.

En estos documentos se visibilizan, con claridad, discusiones y discursos diferenciados entre el hemisferio Norte y América Latina, porque se trata de dos proyectos políticos pedagógicos diferentes. Mientras los primeros están más enfocados hacia una perspectiva ecologista e instrumental, en estas latitudes del sur queda claro que el problema ambiental no es posible resolverlo sin considerar los aspectos sociales, culturales, políticos y económicos. Esta idea ha sido elaborada a través de la ecología política, ver autores como Alimonda (2011), Martínez Alier (1995), Delgado Ramos (2013) y otros. Para solventar la diferencia que se fue explicitando entre desarrollo sostenible y sustentable, los del Norte introdujeron los conceptos de sostenibilidad débil, equivalente a desarrollo sostenible, y sostenibilidad fuerte, equivalente al desarrollo sustentable (GUDYNAS, 2010). 
Por lo tanto, El Decenio de la educación para el desarrollo sostenible (2006) es incentivado y promovido desde la UNESCO con las posiciones del Norte, lo que generó muchas inquietudes y hasta inconformidades en América Latina desde el comienzo, donde se consideró que todos los avances teórico-prácticos logrados en el campo de la educación ambiental aunque inestables y precarios, parafraseando a González-Gaudiano (2011) quien diría -, fueron reemplazados por una conceptualización tan precaria, plana y tendenciosa como la del desarrollo sostenible.

Estas tensiones quedan muy claras con las definiciones que presenta GonzálezGaudiano, quien entiende la educación ambiental:

[...] como práctica pedagógica-política, el proyecto tiende hacia un análisis crítico de la realidad socioambiental en el que prime su transformación en pro de un desarrollo humano responsable. Por el de la educación para el desarrollo sustentable, [como] el proyecto [que] es proclive a salvaguardar los valores y principios de un sistema económico que ha generado la obscena desigualdad mundial existente y una crisis ecológica sin precedente alguno en la historia del planeta. (GONZÁLEZGAUDIANO, 2011, p. 2)

Y añade que la propuesta de la UNESCO trata de un "desplazamiento impuesto desde arriba y afuera que se pretende hacer a través de la educación para el desarrollo sustentable" (GONZÁLEZ-GAUDIANO; PUENTE-QUINTANILLA, 2010, p. 30).

A partir de este contexto histórico-epistémico, considero que el Decenio de Educación para el Desarrollo Sostenible está influenciado por los intereses del Norte para afianzar su discurso del desarrollo. Se trata de un discurso que no vela ni se preocupa por la sustentabilidad por la vida, lo cual se puede demostrar por doquier.

\section{Problematización de la Educación Ambiental Superior en el contexto del desarrollo} sostenible

Ahora, este preámbulo fue importante para contextualizar la educación ambiental superior y los retos que tiene, pero no es objetivo de este artículo evaluar el impacto de este Decenio.

Más bien quiero problematizar la educación ambiental superior dentro de este contexto problemático del desarrollo sostenible, a partir del reconocimiento del poder que tiene el discurso del desarrollo para cooptar la problemática ambiental - como bien lo plantea y visibiliza Escobar (1996) -, facilitado por una episteme de época que despolitiza los instrumentos técnicos y de gestión y ya no cree en partidos ni ideologías políticos, ni tiene proyectos sociales claros; como también considerando que los tomadores de decisiones y ejecutores en torno al discurso del desarrollo, en su gran mayoría, son profesionales formados en universidades, surgen entonces preguntas inquietantes: ¿En qué ha fallado la formación de los profesionales que no logran comprender o asumir la responsabilidad de la complejidad ambiental? ¿Por qué los profesionales universitarios no reconocen la fragilidad de la vida en sus decisiones laborales? ¿Qué tipo de educación ambiental ofrecen las universidades? Se supone, además, que son las ciencias sociales las que deberían ofrecer marcos explicativos y de acción para afrontar los problemas socioambientales, considerando que éstas tienen que ver con decisiones políticas y económicas, con estructuras sociales, con comportamientos culturales etc., que tienen influencias directas sobre el entorno.

Frente a este panorama, es decir, el problema del discurso del desarrollo, así como el problema que tienen las ciencias sociales para comprender la complejidad ambiental, la educación ambiental superior resulta tener un reto aún mayor, por lo tanto requiere de fundamentación teórica sólida para propiciar avances. 
Para dimensionar el problema es necesario reconocer que las ciencias sociales se inscribieron, desde su comienzo, en la modernidad. Una modernidad guiada por el conocimiento del método científico que cosifica y objetiviza al mundo, disociando claramente cuerpo y mente, cultura y naturaleza. Y son estas mismas líneas de pensamiento las que, hoy, impiden comprender la complejidad ambiental. Es decir, aspectos como las condiciones de sustentabilidad ecológicas, en cuanto a su funcionamiento (como p.ej. la resiliencia ecológica y la termodinámica) no han sido consideradas con sus implicaciones de sustentabilidad de la vida, al pensar los procesos productivos y las relaciones sociales. Por lo tanto la política, la economía, la historia o la sociología están inscritas dentro de la racionalidad moderna, que se caracteriza por "su fondo metafísico, su origen mecanicista y organicista, su lógica positivista, su objetivo empirista, su estructura determinista y funcionalista, y su comprensión racionalista que las lleva a desconocer procesos históricos... a sobreexplotar, intervenir y desquiciar el orden ecológico y la diversidad cultural" (LEFF, 2014, p.105).En este contexto, no es posible olvidar que los ejes articuladores de esa modernidad consisten, según Lander (2000, p. 22) en:

1. La visión universal de la historia asociada a la idea del progreso. 2. La naturalización tanto de las relaciones sociales como de la "naturaleza humana" de la sociedad liberal-capitalista. 3. La naturalización u ontologización de las múltiples separaciones propias de esa sociedad. 4. La necesaria superioridad de los saberes que produce esa sociedad ("ciencia") sobre todo otro saber.

Así, esa racionalidad moderna y sus ejes articuladores, conllevan a una manera muy particular y sui generis de conocer el mundo. Un conocimiento secularizado, fragmentado, simplificado y homogeneizante, que pretende ser objetivo, verdadero y universalmente válido, y que ha desplazado las preguntas del ¿Por qué? o ¿Esto es bueno? por las preguntas del ¿Cómo? y ¿Esto funciona? Todo debe ser Zweckrational y el mundo se convierte así en "una colección de materia inerte que se mueve rápidamente sin fin ni significado" (BERMAN 2001, p. 45), materia para ser medida, para ser predecible, planificable y explotable.

$\mathrm{Al}$ concebir desde las bases mismas de las formas de conocer una escisión profunda entre cultura y naturaleza, como ya lo evidencia la categoría de naturalista de Descola (2012), resulta difícil, y casi imposible, comprehender las interrelaciones e interdependencias que existen en y sobre el planeta vivo. Es así, entonces, como p.ej. las teorías económicas, preocupadas por las formas de organización de la producción y su crecimiento para obtener riquezas materiales, se piensan totalmente desligadas de las condiciones ecológicas y termodinámicas de la naturaleza. Y la sociología, preocupada por la organización de las estructuras sociales y el progreso, se consolida a través de "tres principios fundamentales: la influencia del positivismo como esquema lógico, el funcionalismo como método, la concepción de la "sociedad industrial" como contexto y la "teoría de la modernización" como marco de inteligibilidad" (LEFF, 2014, p. 34). Enfoques profundamente arraigados en las disciplinas y las facultades, que son sistemáticamente producidas y reproducidas en las universidades, tanto en la investigación como en la formación.

Ahora, antes de hablar de la educación ambiental superior propiamente dicha, es necesario aclarar que, por un lado, ésta no se puede instrumentalizar para solucionar problemas concretos de contaminación o realizar un ordenamiento territorial, entre otros, a través de proyectos de intervención, ni para realizar jornadas de reforestación o reciclaje en las universidades, ni para promover educación ambiental en las comunidades aledañas de la universidad, como a veces se observa. Y, por el otro lado, tampoco está para solucionar esos problemas profundos epistemológicos y filosóficos mencionados, en este caso de las propias ciencias sociales (ya que, en ese caso, serán ellas mismas, a través de proyectos de investigación las que tendrán que ir solventándolos).

También es de señalar que en la educación ambiental superior no solo se trata de generar una conciencia ambiental o de llenar a los estudiantes de información sobre tecnologías, 
legislación, y normas e instrumentos de políticas ambientales, sino más bien de relacionar e identificar directamente, desde cada disciplina, cómo se relaciona cada una con su entorno y con la vida. Es decir, se trata más bien de proveer a los futuros profesionales de un panorama lo suficientemente amplio para comprender la complejidad ambiental. Esto significa que cada disciplina se tendrá que revisar y evidenciar de qué manera sus conocimientos, sus formas de concebir a la naturaleza, y por ende sus formas de intervenir en la sociedad o de transformar el territorio, afectan, o no, la sustentabilidad de la vida a largo plazo sobre el planeta.

Por lo tanto, sin pretender dar una definición perfecta, se puede entender la educación ambiental superior como aquella que ofrece una formación ambiental a los futuros profesionales para que sean capaces de comprender las interrelaciones e interdependencias entre sociedad y naturaleza (lo cual implica comprender la complejidad y sensibilidad de los ecosistemas), entre visiones de mundo y formas de transformación del territorio, de comprender las implicaciones socioambientales de la racionalidad económica e instrumental hegemónica, que no consideran las condiciones de sustentabilidad de la vida en la producción (como p.ej. la entropía y la resiliencia) y reconocer el poder en el saber, para relacionar todos estos conocimientos, desde y con cualquiera de las disciplinas en las que se estén formando.

En suma, se trata de ofrecer a los futuros profesionales criterios contextuales y teóricos sólidos, amplios y comprehensivos para tomar decisiones ambientales responsables, reconociendo sus implicaciones ambientales a largo plazo. Este es el gran reto que tiene la educación ambiental superior. Porque significa que definitivamente, no serán suficientes las materias aisladas o la trasmisión de instrumentos ambientales, sino que se requiere de cambios profundos y comprometidos de un grupo mayor de profesores, dispuestos a debatir, discutir, construir y a cambiar el paradigma moderno dominante, lo cual, en últimas, significa también trascender los límites disciplinarios, pasar a la interdisciplinariedad y más allá de la transdisciplinariedad. Tal vez por la misma razón, esta tarea ha resultado ser tan difícil, y de que algunos hablen de las universidades blindadas o como baluartes ante el tema ambiental, porque cada disciplina, cada grupo de investigación, cada profesor termina velando por sus intereses y trayectorias disciplinarias, manteniéndose en lugares cómodos. Pero el saber ambiental implica cuestionar, dislocar, incomodar, repensarse creativamente.

La educación ambiental debe evidenciar y develar la relación directa que existe entre las diferentes conceptualizaciones de ambiente y sus efectos concretos sobre el espacio y el territorio. Así, por ejemplo, la propuesta de tres categorías de ambiente que hago, puede facilitar este trabajo. Ambiente como objeto, que se fundamenta en una epistemología positivista, ambiente como sistema, que se fundamenta en una epistemología sistémica (no compleja adaptativa), y ambiente como crítica a la visión dominante, que se fundamente en la complejidad y el saber ambiental (ESCHENHAGEN, 2009). Cada categoría propondrá una educación ambiental con perspectivas específicas y, por ende, con implicaciones diferentes sobre el entorno a largo plazo, que no siempre son evidentes a primera vista.

Al diferenciar estas tres categorías de ambiente por sus bases epistemológicas es más fácil reconocer qué tipo de educación se está proponiendo, ya que no es lo mismo enseñar desde una epistemología positivista que desde la complejidad. Estas tres categorías pueden, además, ayudar por un lado a visibilizar la diversidad de ofertas, posiciones y propuestas para comprender y afrontar el problema ambiental, y, por el otro, a fortalecer las propuestas de educación ambiental, para proporcionar un marco epistemológico más sólido.

Definitivamente, una tarea central de la educación ambiental superior es mostrar las múltiples posiciones posibles que se pueden adoptar dentro de la educación ambiental. Cada una tiene puntos de partida diferentes, con epistemologías diferentes que las soportan y que se mueven dentro de diversos paradigmas, discursos e intereses. Hoy se hace cada vez más urgente entre los educadores ambientales enfrentarse a una discusión tanto paradigmática como epistemológica para poder enfrentar, eficazmente, los retos de ésta época en crisis. Uno de los 
elementos de esta discusión debe proponer una reflexión sobre la aceptación o no de una educación ambiental para el desarrollo sostenible. Esta discusión tiene que aclarar cuáles son las implicaciones y los problemas neurálgicos en todas sus dimensiones (políticas, económicas, culturales, sociales, ambientales etc.).

La educación ambiental tendrá, entonces, que adquirir la capacidad, a través del saber ambiental, de visibilizar con más claridad las in-coherencias entre los discursos y las prácticas que se observan por doquier en la sociedad. De ahí también la necesidad de preguntarse por los puntos de partida para repensar la educación ambiental superior, lo cual claramente está inmerso en el problema de los discursos del desarrollo y requiere de un pensamiento ambiental, capaz de proporcionar criterios y directrices.

El reto consiste, entonces, en posibilitar un pensamiento ambiental que haga comprensible por qué y cómo somos hoy, aquello en lo que hemos devenido como sociedades modernas, es decir, el reto está en evidenciar la relación que existe entre las formas de pensar y el manejo y la apropiación que se le da al entorno natural. En este contexto también es importante plantearse preguntas como: ¿Por qué se niega, oculta o desdibuja, a través de velos ideológicos y paradigmas científicos, la relación entre los problemas ambientales y el sistema económico (y, más específicamente, el consumo y las necesidades)? ${ }^{4}$ ¿Por qué es tan difícil comprender la estrecha interdependencia del ser humano con la naturaleza? ¿Cómo salir/superar este sistema complejo altamente adaptativo, pero insustentable, que resulta ser el capitalismo? Claramente estas preguntas no se pueden responder de manera fácil, ni inequívocamente, más bien son preguntas latentes, con múltiples entradas y cuyas respuestas reflejarán diferentes posturas cosmológicas y epistemológicas. Éstas, a la vez, permitirán aproximarse a la comprensión de las raíces de los problemas ambientales y así proponer soluciones posibles. Plantear este tipo de preguntas debería ser, además, una característica central del pensamiento ambiental y en la formación de los futuros profesionales.

Dentro de este contexto, la educación ambiental consiste, por un lado, en desplegar y discutir todas las aristas del pensamiento ambiental para comprender la complejidad ambiental, $\mathrm{y}$, por el otro, en capacitar para vivir en un mundo cambiante sin destruir las estructuras ecológicas que posibilitan la propia vida. Es decir, proporcionar el conocimiento necesario para poder comprender, contextualizar y transformar las actitudes, los comportamientos, las formas de producción, las relaciones de poder y los paradigmas dominantes que han resultado ser nocivos tanto para la convivencia social como para el entorno. Vivir en un mundo cambiante implica, también, proporcionar las capacidades necesarias para construir un proyecto de futuro, un proyecto que se atreva a pensar por fuera de los paradigmas que imperan, y mostrar que otros mundos son posibles mientras se posibiliten culturas alternativas, con valores, éticas y estéticas que permitan una convivencia viable a largo plazo entre los seres humanos, sociedades y culturas, y entre los humanos y la naturaleza.

Esto significa ambientalizar la educación ambiental, partir de la tercera categoría de ambiente, como crítica a la visión dominante, pues: ¿Cómo se puede enfrentar un problema si no se conocen sus causas profundas y complejas? Si las causas no se consideran, pueden surgir propuestas de acción que solamente actúan de manera instrumental, superficial y puntual. ¿Cómo hablar de educación ambiental si no se recupera el respeto por la vida? Es necesario comprender la complementariedad y la interdependencia así como reconocer la importancia de la diversidad y la diferencia. Se requiere de un principio de responsabilidad que considere las consecuencias temporales y geográficas del actuar. Y, finalmente, ¿Cómo enfrentar estos retos si no se incentiva lo cognitivo lo sinérgico, simultáneo, intuitivo al reconocer que lo analítico,

\footnotetext{
${ }^{4}$ Un ejemplo claro sería el caso del cambio de la matriz energética en Alemania, muy preocupada por salir de la energía atómica, pero muy raras veces se encuentran discusiones o cuestionamientos sobre el por qué y para qué se necesita tanta energía.
} 
atomista, racional no son suficientes? El desafío está en buscar caminos para superar la estrechez e insustentabilidad de las formas de conocer modernas.

Definitivamente, no hay una receta o herramienta, ni un camino inequívoco para decir esto es la educación ambiental superior. Tampoco, se trata de simplemente incorporar la dimensión ambiental a los temas y la educación u ofrecer una materia adicional sobre lo ambiental (ESCHENHAGEN, 2010). Esto justamente hace tan difícil la educación ambiental superior si se concibe y piensa el ambiente como un concepto capaz de replantear y reconstruir el conocimiento desde la complejidad ambiental y no como un objeto.

El mundo hegemónico moderno se ha incorporado, de manera profunda y sistemática, en el pensar y actuar cotidiano, y se expresa a través de los presupuestos de la racionalidad instrumental y economicista, así como del conocimiento fragmentado. Estos últimos están presentes en la toma de casi cualquier decisión tanto pública como privada. Es una cosmovisión profundamente incorporada, naturalizada y arraigada en el ser y actuar cotidiano. Por lo tanto, es necesario revisar, comprender e ir más allá de esta visión hegemónica y construir visiones alternas desde la complejidad ambiental, que terminen permeando el ser y habitar de la sociedad y las personas para vivir y habitar de otras maneras.

Por lo tanto, frente a esta cosmovisión moderna que dificulta comprender la complejidad ambiental, la educación ambiental superior exige, primero, una comprensión y desnaturalización de ésta, que se traduce en una epistemología ambiental, a partir de la cual sea posible habitar de manera sustentable este planeta. Que permita un habitar y convivir, ya no a partir de los supuestos hegemónicos de la racionalidad instrumental y económica, el conocimiento fragmentado, sino a partir de la racionalidad ambiental. Así, planteo cuatro elementos centrales que debe considerar y contener una educación ambiental superior:

1. Evidenciar y reconocer la relación que existe entre formas de conocer y sus modos de justificar la apropiación y adaptación al entorno natural de las diferentes sociedades.

2. Recuperar los aportes que puede dar la filosofía para pensar y reconocer el papel de las distintas disciplinas y su quehacer y responsabilidad en una sociedad. Es decir, recuperar la filosofía para pensar.

3. Reconocer que la visión de mundo hegemónica está atravesada por relaciones de poder y fundamentada por la racionalidad moderna cientificista, que impide comprender la complejidad ambiental, ésta debe ser problematizada, evidenciada y demostrada, a través del saber ambiental.

4. Revisar sistemáticamente las bases epistemológicas de las respectivas disciplinas y sus supuestos respecto a la naturaleza, para visibilizarlas y transformarlas en y para la producción y reproducción del conocimiento en la formación de los futuros profesionales.

5. Propiciar el conocimiento para comprender las relaciones que existen entre los factores bióticos y abióticos, y conocer los diferentes ciclos geo, bio y ecosistémicos (trófico, agua, carbón, fósforo etc.) y comprender las implicaciones de la termodinámica, la entropía etc., es decir, aproximarse también a las ciencias de la complejidad $^{5}$

6. Fomentar la capacidad de asombro, curiosidad y creatividad, sin los cuales serán imposibles alternativas otras.

La educación ambiental superior, enfocada específicamente en la formación de los futuros profesionales, consiste, entonces, en la capacidad de los profesores de poder articular de estos puntos, a través del diálogo de saberes, para construir una cosmovisión desde la cual sean posibles otras formas de ser, estar y habitar el mundo, y que respeten la diversidad de

${ }^{5}$ Ver por ejempo: Instituto Santa Fe: <http://www.santafe.edu/>, New England Complex Systems Institute (NECSI): <http://necsi.edu/> 
formas de vida. Significa ofrecer en su conjunto elementos para formar criterios ambientales para decisiones futuras en las profesiones futuras. Es de señalar, además, que en la medida que se adquiere este conocimiento, a través de la capacidad de asombro, de conmoverse y la creatividad, se logra una capacidad de reflexión crítica-constructiva, ligada al reconocimiento de la responsabilidad ética que se obtiene con el conocimiento para habitar el mundo. El fin último consiste en posibilitar y garantizar, a largo plazo, la reproducción de la vida misma.

\section{Referencias}

ALIMONDA, H. (Coord.). La naturaleza colonizada, ecología política y minería en América Latina. Buenos Aires: CICCUS,/CLACSO, 2011.

ÁNGEL MAYA, A. La fragilidad ambiental de la cultura. Bogotá: Universidad Nacional de Colombia, 1995.

ÁNGEL MAYA, A. El reto de la vida. Ecosistema y cultura, una introducción al estudio del medio ambiente. Bogotá: ECOFONDO, 1996.

ARIAS, M. Á. Desarrollo sustentable: una propuesta ante la desilusión del progreso. En: Portada de la Academia Nacional de Educación Ambiental del México [Online]. Ciudad del México: ANEA, 2003. Disponible en: <http://www.anea.org.mx/docs/Arias-DesilusionProg.pdf>. Acceso en: 16 ago. 2016.

BERMAN M., El reencantamiento del mundo. Santiago: Cuatro Vientos, 2001.

CAVALCANTI, C. Uma tentativa de caracterização da economia ecológica. Ambiente \& Sociedade, Brasil, v.7, n.1, p. 149-156, Jan./Jun. 2004. Disponible en: <http://www.scielo.br/pdf/\%0D/asoc/v7n1/23541.pdf>. Acceso en: 16 ago. 2016.

CORTÉS LANDÁZURY R. Tensión o revolución en la economía del medio ambiente?: Un balance crítico. Economía Gestión Desarrollo, Cali, n.6, p.165-189, Dic., 2008. Disponible en: <http://uniciencia.ambientalex.info/infoCT/Revolucioneconomica.pdf>. Acceso en: 30 ago. 2016.

DELGADO RAMOS, G. C. (Coord.) Ecología política del extractivismo en América Latina: Casos de resistencia y justicia socioambiental. Buenos Aires: CLACSO, 2013. Disponible en: <http://biblioteca.clacso.edu.ar/clacso/posgrados/20131218030905/EcologiaPolitica.pdf>. Acceso en: 30 ago. 2016

DESCOLA, P. Más allá de naturaleza y cultura. Buenos Aires: Amorrortu, 2012.

ESCHENHAGEN, M. L., Educación ambiental superior en América Latina, Retos epistemológicos y curriculares. Bogotá: UDCA/ECOE, 2009.

ESCHENHAGEN, M. L. Aportes ambientales desde América Latina para la apertura de las Ciencias Sociales. Bogotá: Universidad Central, 2010.

ESCHENHAGEN, M. L. Contexto y exigencias a las ciencias sociales para afrontar los problemas ambientales. Polis Revista Latinoamericana, Santiago, n.30, p. 1-17, Ene. 2012. Disponible en: <http://polis.revues.org/2341>. Acceso en: 30 ago. 2016.

ESCHENHAGEN, M. L., 2015, Desafíos para pensar desde la vida en las ciencias sociales. Polis Revista Latinoamericana, Santiago, n.41, p. 1-10, Sept. 2015. Disponible en: <http://polis.revues.org/10909>. Acceso en: 30 ago. 2016. 
ESCOBAR, A. La invención del tercer mundo, construcción y deconstrucción del desarrollo. Bogotá: Norma, 1996.

GALAFASSI, G. P. Las preocupaciones por la relación naturaleza-cultura-sociedad. Ideas y teorías en los siglos XIX y XX. Una primera aproximación. Revista Theomai, Buenos Aires, n. 3, p. 1-9, primer semestre. 2001.

GALAFASSI, G. P. Ambiente, sociedad y naturaleza, entre la teoría social y la historia. Buenos Aires: Universidad Nacional de Quilmes, 2002.

Disponible en: 〈http://www.redalyc.org/articulo.oa?id=12400311〉. Acceso en: 30 ago. 2016.

GONZÁLEZ-GAUDIANO E. J.; PUENTE-QUINTANILLA, J. C. El perfil de la educación ambiental en América Latina y el Caribe: Un corte transversal en el marco del Decenio de la Educación para el Desarrollo Sustentable. Revista Pesquisa em Educação Ambiental, Rio Claro, v.5, n.1, p. 27-45, primer semestre. 2010. Disponible en: 〈http://www.revistas.usp.br/pea/article/view/30084>. Acceso en: 30 ago. 2016.

GONZÁLEZ-GAUDIANO, E. La educación ambiental en américa latina: rasgos, retos y riesgos. Revista Contrapontos, Itajaí, v.11, n.1, p. 83-93, Jan./Abr. 2011.

GRANATO, L.; NAHUEL, O.; PENELA, A. C. Valoración económica del medio ambiente: "las propuestas de la economía ecológica y la economía ambiental. Revista OIDLES, Malaga, v.3, n.7, s/p, Dic. 2009. Disponible en: 〈http://www.eumed.net/rev/oidles/07/go.htm〉. Acceso en: 30 ago. 2016.

GUDYNAS, E. Desarrollo sostenible: una guía básica de conceptos y tendencias hacia otra economía. Otra Economía, Buenos Aires/São Leopoldo, v.3, n.6, p.43-66, 1er Sem., 2010.

LANDER, E.. Ciencias sociales: saberes coloniales y eurocéntrico. (Comp.). La colonialidad del saber: eurocentrismo y ciencias sociales. Perspectivas Latinoamericanas. Buenos Aires: CLACSO, 2000 .

LEFF, E. Racionalidad ambiental, la reapropiación social de la naturaleza. México: Siglo XXI, 2004.

LEFF, E. Aventuras de la epistemología ambiental. De la articulación de las ciencias al diálogo de saberes. México: Siglo XXI, 2006.

LEFF, E. La apuesta por la vida. México: Siglo XXI, 2014.

MARTINEZ-ALIER, J., De la economía ecológica al ecologismo popular. Barcelona/Montevideo: Icaria/Nordan, 1995.

NACIONES UNIDAS, Agenda 21. Disponible en: <http://www.famp.es/recsa/Documentos/2_Agenda_21/A_Agenda21_Local.pdf>. Acceso en: 30 ago. 2016 .

ORGANIZACIÓN DE ESTADOS IBERO-AMERICANOS - OEI. Carta de la Tierra - 2000. Disponible en: 〈http://oei.es/salactsi/charter_es.pdf〉. Acceso en: 30 ago. 2016.

ORGANIZACIÓN DE LAS NACIONES UNIDAS - ONU. Informe Brundtland 1987. Disponible en: <http://www.un.org/es/comun/docs/?symbol=A/42/427>. Acceso en: 30 ago. 2016.

ORGANIZACIÓN DE LAS NACIONES UNIDAS PARA LA EDUCACIÓN, LA CIENCIA Y LA CULTURA. Decenio de las Naciones Unidas de la Educación con miras al Desarrollo Sostenible 

(2005-2014): Plan $\quad$ de $\quad$ aplicación $\quad$ internacional.
<http://unesdoc.unesco.org/images/0014/001486/148654so.pdf>. Acceso en: Acceso en: 30 ago. 2016.

PROGRAMA DE LAS NACIONES UNIDAS PARA EL MEDIO AMBIENTE - PNUMA. Manifiesto por la vida: por una ética para la sustentabilidad. Disponible en: < http://www.uci.ac.cr/descargas/VICDESARROLLO/DESARROLLO-SOSTENIBLE/Conveciones-protocolosdocumentos/Otros/Manifiesto-por-la-Vida.pdf>. Acceso en: 16 ago. 2016. 